reduced survival on univariable analysis. Both of these associations remained significant on multivariable analysis correcting for demographic variables, disease severity (as each of: CPI, FVC, and DLCO), and treatment. The association of both measures of decline with early mortality were also maintained when, in separate models, PASP $\geq 40 \mathrm{~mm} \mathrm{Hg}$ on echocardiography, and BAL lymphocytosis thresholds of $20 \%, 30 \%$ and $40 \%$, were included in addition to demographic variables, disease severity, and treatment.

Conclusions A marginal worsening in FVC of $\geq 5 \%$ and in DLCO of $\geq 10 \%$ at one year are predictive of markedly reduced survival in $\mathrm{fHP}$.

\section{P146 PREDICTORS OF ADVERSE OUTCOME IN SARCOIDOSIS COMPLICATED BY PULMONARY ASPERGILLOSIS}

${ }^{1} \mathrm{~L}$ Nwankwo, ${ }^{2} \mathrm{~J}$ Periselneris, 'D Gilmartin, ${ }^{1} \mathrm{~S}$ Desai, ${ }^{1} \mathrm{~A}$ Shah, ${ }^{1} \mathrm{~V}$ Kouranos, ${ }^{1} \mathrm{AU}$ Wells, ${ }^{1} \mathrm{E}$ Renzoni, ${ }^{1} \mathrm{PL}$ Molyneaux, ${ }^{1} \mathrm{P}$ George, ${ }^{1} \mathrm{M}$ Kokosi, ${ }^{1} \mathrm{~A}$ Devaraj, ${ }^{1} \mathrm{D}$ Armstrong-James, ${ }^{1}$ F Chua. ${ }^{1}$ Royal Brompton Hospital, Guy's and St. Thomas' NHS Foundation Trust, London, UK; ${ }^{2}$ Kings College Hospital, London, UK

\subsection{6/thorax-2021-BTSabstracts. 255}

Introduction Aspergillosis complicating pulmonary sarcoidosis is associated with high mortality. The specific prognostic impact of fibrocavitary sarcoid disease, however, remains poorly understood. A better understanding of the factors that determine adverse outcomes in such patients may improve the management of both the underlying sarcoid disease and associated secondary fungal infection.

Methods Cases of pulmonary sarcoidosis with elevated Aspergillus IgG (>40 mgA/L) presenting between January 2009 and March 2021 were retrospectively identified. Controls (sarcoidosis with normal Aspergillus IgG titre) were case-matched by baseline $\%$-predicted gas transfer factor (TLco; $\pm 5 \%$ variance). Computed tomography (CT), baseline lung function and survival data were analysed.

Results Among 180 cases (high Asp IgG) and 229 controls (normal Asp IgG), no inter-group difference was evident in the median age at presentation [48 (IQR 40-58) vs 50 (IQR 42-59)] or gender (proportion female: $45.5 \%$ vs $51.1 \%$ ). Amongst the cases, 81/180 (45\%) had fibrocavitary changes, compared with $14 / 229(6.1 \%)$ of the controls $(\mathrm{P}<0.001)$. Radiologically-evident aspergilloma was present in $79 \%$ (64/ 81 ) of the cases with fibrocavitary sarcoidosis. These cases also had poorer lung function compared to non-fibrocavitary disease: mean $\%$-predicted forced vital capacity/FVC $71.3 \%$ vs

\section{Survival proportions}

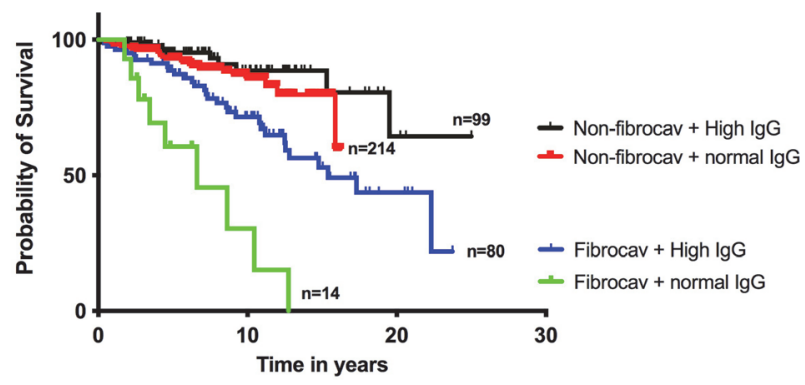

Comparison of survival curves by log-rank, $p$ value $=<0.0001$

Abstract P145 Figure 1 Survival proportions
91.4\% $(\mathrm{P}<0.0001)$, and in the controls: $69.9 \%$ vs $89.1 \%$ $(\mathrm{P}<0.01)$. A similar trend was observed in $\%$-predicted TLco amongst the fibrocavitary cases: $45.8 \%$ vs $64.2 \%$ non-fibrocavitary $(\mathrm{P}<0.0001)$ and in the fibrocavitary controls: $39.7 \%$ vs $61.8 \%$ non-fibrocavitary $(\mathrm{P}<0.0001)$. Comparing only those with fibrocavitation, neither the $\%$-predicted FVC $(71.3 \%$ vs $69.9 \%$ predicted; $\mathrm{P}=0.82)$ nor $\%$-predicted TLco $(45.7 \%$ and $39.7 \%$ predicted; $\mathrm{P}=0.17$ ) differed between cases and controls. Evidence of fibrocavitary destruction was associated with higher overall mortality (37\% vs $9.1 \%$ in the non-fibrocavitary subgroup; <0.0001) and longest median survival (graph).

Conclusions Fibrocavitary sarcoidosis is associated with worse lung function and poorer median survival. In this group, elevated Aspergillus IgG highlights a greater incidence of aspergilloma.

Implications Fibrotic transformation of pulmonary sarcoidosis heightens symptom burden, predisposes to chronic Aspergillus infection and is prognostically important particularly when there is supervening fibrocavitary lung destruction. Sensitive stratification of such patients for long-term outcome may help identify particular individuals for earlier and more focused therapeutic intervention.

\section{P147 LONG-TERM PULMONARY FUNCTION AND MORTALITY OUTCOMES IN IDIOPATHIC PULMONARY FIBROSIS PATIENTS TREATED WITH ANTIFIBROTICS}

J Barnes, E Harris, L Matos, K Harding, M Thillai. Royal Papworth Hospital NHS Foundation Trust, Cambridge, UK

\subsection{6/thorax-2021-BTSabstracts.256}

Background Idiopathic Pulmonary Fibrosis (IPF) is a progressive scarring lung disease. The antifibrotics pirfenidone and nintedanib are approved for IPF patients with a forced vital capacity $\%$ predicted (FVC\%) between 50-80\%, and either drug may be prescribed in the first instance. Both drugs reduce mortality risk, and disease progression as assessed by FVC $\%$ and transfer factor for carbon monoxide $\%$ predicted $\left(\mathrm{TL}_{\mathrm{CO}} \%\right)$ over 12-months. The effectiveness of antifibrotics beyond 12-months is less established, furthermore there is limited real world data comparing pulmonary function and mortality between both drugs.

Aims To assess the effectiveness of antifibrotics on $\mathrm{FVC} \%$ and $\mathrm{TL}_{\mathrm{CO}} \%$ over 24-months. To identify if the current clinical rationale of offering either antifibrotic from the outset is appropriate through investigating $\mathrm{FVC} \%, \mathrm{TL}_{\mathrm{CO}} \%$ and mortality outcomes in IPF patients receiving pirfenidone or nintedanib.

Methods We carried out a retrospective analysis of IPF patients with an FVC\% between 50-80\% who commenced antifibrotic treatment between May 2012 and October 2019 at Royal Papworth Hospital (Cambridge, UK). Separate random coefficient regression models were used to assess $\mathrm{FVC} \%$ and $\mathrm{TL}_{\mathrm{CO}} \%$ at $0,6,12,18$ and 24 -months. A Cox proportional hazards model was used to assess hazard ratio (HR) and 95\% confidence interval (CI) for all-cause mortality in antifibrotic treated patients.

Results 194 patients were identified from our dataset. FVC $\%$ remained stable between 0 and 12 -months $(\mathrm{p}=0.330)$ but declined between 12 and 24-months $(\mathrm{p}<0.001)$. $\mathrm{TL}_{\mathrm{CO}} \%$ decreased between 0 and 12-months $(p=0.003)$ and between 12 and 24 -months $(\mathrm{p}<0.001)$. Over 24 -months, FVC\% was 


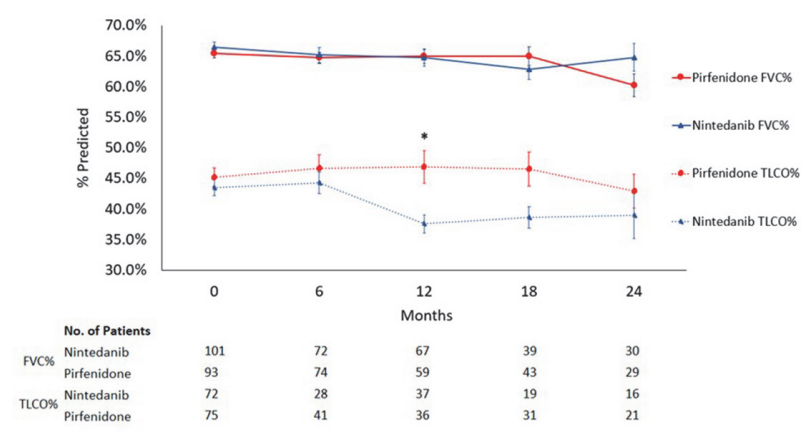

Abstract P147 Figure 1 Mean FVC\% (Block lines) and $\mathrm{TL}_{\mathrm{CO} \%}$ (Dotted lines) over 24-months in patients treated with pirfenidone (Circle) and nintedanib (Triangle). * Significant difference between nintedanib and pirfenidone

similar between pirfenidone and nintedanib (Figure 1) $(\mathrm{p}=0.495)$, while $\mathrm{TL}_{\mathrm{CO}} \%$ was greater in pirfenidone treated patients (figure 1$)$, albeit non-significant $(p=0.054)$. No allcause mortality difference was observed for nintedanib versus pirfenidone $(\mathrm{HR}=0.80 \quad[95 \%$ CI $=0.46-1.40]$; $\mathrm{p}=0.434)$.

Conclusions Data from our centre revealed a greater FVC\% decline during the 2 nd year of treatment compared to the 1 st year of treatment. There appears to be no difference in pulmonary function parameters and all-cause mortality between pirfenidone and nintedanib. This supports the current clinical rationale that if there are no contraindications to either drug, patients should be offered the choice of antifibrotic at the outset.

\section{\begin{tabular}{|l|l}
\hline P148 THE COMORBIDOME OF SARCOIDOSIS \\
\hline
\end{tabular}}

S Gilmour, M Donaghy, P Minnis, E Murtagh. Interstitial Lung Disease Service, Antrim Area Hospital, Northern Health and Social Care Trust, Antrim, UK

\subsection{6/thorax-2021-BTSabstracts.257}

Introduction and Objectives The concepts of comorbidity and polypharmacy are emerging as important factors in the evaluation, risk stratification, and treatment of patients. As in other diseases, typical of middle-aged and elderly individuals, patients with Sarcoidosis are often affected by one or more comorbidities. The BTS registry currently records TB, current malignancy, Diabetes, Hypertension and Ischaemic Heart Disease as comorbidities in sarcoidosis. We aimed to explore a large cohort to assess prescription trends, identify comorbidity and possible treatment phenotypes.

Methods We identified 151 patients with a tissue confirmed diagnosis of sarcoidosis attending a dedicated clinic and reviewed their electronic care record for filled prescriptions within 3 months. Medications prescribed were used to identify polypharmacy as well as a surrogate marker for co-morbidity (due to coding inefficiencies). Medications were grouped according to indication including treatment need, cardiovascular, metabolic, gastroenterological, musculoskeletal, and psychological disease. We calculated the prevalence of co-morbidities and modelled associations.

Results The 151 patients identified in the cohort included 84 males and 67 females, with an average age of 56. 1065 medications were prescribed, with an average of 7.05 tablets per patient. $44 \%$ of patients are on sarcoidosis treatment with
$67 \%$ of these managed on single therapy, $28 \%$ on double therapy and $4 \%$ on triple therapy. $4 \%$ of patients are prescribed Imraldi. Interestingly, the average number of tablets prescribed increases in the treatment group to 8.8, despite age and gender not being significantly altered. When the medications were grouped the most common prescription groups included gastrointestinal protection, analgesia and bone protection. In keeping with recognised co-morbidities $50 \%$ of patients are on primary or secondary cardiac preventative therapy. Comparing patients on treatment with the general cohort there is an increase in the number of patients prescribed analgesia (56\% compared with 44\%) and antidepressants and antipsychotics (34\% compared with 29\%).

Conclusion The pattern and impact of comorbidities give significant insights to treatment phenotypes of sarcoid patients attending clinic and inform treatment choices. This granular approach lends itself to Precision Medicine allowing the customization of healthcare being tailored to a subgroup of patients, instead of a one drug fits all approach.

\section{P149 INCIDENCE AND PREVALENCE OF LEFT-SIDED HEART FAILURE IN PATIENTS WITH IDIOPATHIC PULMONARY FIBROSIS: A POPULATION-BASED STUDY}

${ }^{1,2} \mathrm{~A}$ Koteci, ${ }^{1,2} \mathrm{AD}$ Morgan, ${ }^{1,2} \mathrm{HR}$ Whittaker, 1,2 L Portas, 1,3PM George, 1,2,3 JK Quint. ${ }^{1}$ National Heart and Lung Institute, Imperial College London, UK; ${ }^{2}$ NIHR Imperial Biomedical Research Centre, London, UK; ${ }^{3}$ Royal Brompton Hospital, London, UK

\subsection{6/thorax-2021-BTSabstracts.258}

Background In absence of large epidemiological studies, it is unknown how common left-sided heart failure (HF) is in patients with idiopathic pulmonary fibrosis (IPF), and whether incidence and prevalence has changed over time.

Methods Patients aged $>40$ years with IPF were included from the Clinical Practice Research Datalink Aurum database of nationally-representative, de-identified primary-care electronic healthcare records covering $18 \%$ of the UK population. $\mathrm{HF}$ prevalence and incidence were calculated yearly over 10 years (1 January 2010-31 December 2019), stratified by age and sex.

Results 25, 341 IPF patients were included. Median age was 76.4 years (IQR 68.7-82.5). Between 2010-2019, prevalence of HF decreased from 33.4\% (95\% CI 32.2-34.6) in 2010 to $20.9 \%(20.0-21.7)$ in 2019. At all time-points, prevalence was higher in men than women, and with increasing age (in 2019: $8.2 \%(6.2-10.7)$ in those aged $40-59 ; 16.2 \%$ (15.1$17.3)$ in those aged 60-79; and 28.8\% (27.3-30.2) in those aged >80). HF incidence rate per 100 person-years (95\% CI) remained stable between 2010-2017 but decreased from 4.3 (3.9-4.8) in 2017 to $3.4(3.0-3.9)$ in 2019. This trend was noted in both sexes and different age groups.

Conclusion While prevalence of HF in IPF is consistent with previously published estimates and similar to COPD cohorts, incidence rate is 3.9 times higher in IPF than COPD patients at comparable timepoints. ${ }^{1}$ While decreasing HF incidence is seen in both IPF patients and the general population, decreasing prevalence in the former contrasts stable prevalence in the latter. ${ }^{2}$ Decreasing HF incidence in IPF patients could reflect improved management of cardiovascular risk factors, while decreasing prevalence may be secondary to high mortality. Further studies should help explore factors driving the trends observed and aetiology of HF in IPF. 